\title{
Research on Personalized and Secured E- Learning Systems using Data Mining Technique
}

\author{
G.Babu, C. Thirumal Azhagan
}

\begin{abstract}
Data mining is the concept for extracting the appropriate data from the large set of database. In today's world it is widely used for many applications where learning applications is one of the major part. The e-Learning is the booming technology where anyone can learn everything from any part of the world. It is the digital way of learning the concepts and does not require the help of other persons to do so. It also requires the large space for data storage such as user information, course records and course details and so on. There are lot of learning applications available on the internet among which some might be subjected to frauds. So the security is the demanding thing every users looking for to protect their details. The users also seek for flexibility of using the applications. In perspective of distributed world, the complexity and interoperability of the data brings challenges in e-learning domain.Depends upon learner's choice, the web based learning modules were developed for the students. Thus, a holistic approach is required for achieving the personalized content since the student groups are heterogeneous in nature. In addition to, the personalized content has to be protected in order to maintain the data integrity and privacy of the users. In this work, we survey about the present scenario of the web-based e-learning systems. Initially, we present the services oriented architecture of the e-learning systems and also clearly explain the different $e$ learning layers.Then, we portray the existing studies processed in web based e-learning systems. Finally, we discuss about the challenges still persists in web-based learning systems. This paper will guide the upcoming researchers in e-learning fields.
\end{abstract}

Keywords: E-learning systems, Service Oriented Architecture, Personalization, Security and Interoperability.

\section{INTRODUCTION}

The recent developments made in communication technologies has introduced in the educational systems which is coined as "e-Learning systems". With the use of technologies, the concept of e-learning is defined.The services like delivery, administration and the assessment of the learning process.It's a two way communication that encourages the students for limited period [1]. The perception of resources is done by the learners.Irrespective of the location, the students can complete the courses.By doing so, the interface designers design different learning models. Success is the main factors of the e-learning systems. Several group of researchers assists to design an effective course materials. This sorts of design helps to increase the success rate of the systems [2].

The factors like learning via technologies and drop outs from various courses have helped the students. Different

Revised Manuscript Received on September 10, 2019.

Dr. G. Babu, Professor, Department of Computer Applications, Adhiparsakthi Engineering College, Melmaruvathur, Tamilnadu, India.

(email id: babughi@gmail.com)

Dr. C. Thirumal Azhagan, Assistant Professor,Department of Management Studies, Anna University, BIT Campus Trichy, Tamilnadu, India.

(email id: azhaganct@gmail.com) students inherit different characteristics which degrades the design of the courses. Recently, personalized e-learning system attracts the students. Since itmakes use of online environment, the students personalize their learning environment. The personal and pedagogical choices of the students assist to design the courses. It is also inferred that group of students are heterogeneous in nature.It is also important that the heterogeneity factors of the student differentiate the groups. Therefore, holistic model is developed for each group of students from different environment. Conventional teaching models didn't support the different levels of students. Different learning systems improve the learning process among different people $[4,5]$.

Personalization is a theme which develops trusted environment for better learning models [6].The system maintains its own infrastructure process. The modification in learning path is done from perspective of the students [7, 8].The advancement made in learning management systems surveyed the performance of the learners. Based on requirement of the learner's opinion, the learning activities are designed.In LMS, properties of content are not easily perceptable. The learning design of the content and its properties are well equipped for different learners. Some of the semantic features are also intrigued for learning modalities [9].

\section{SUREVEY LITERATURE}

The main task of SOA is to design a distributed system that integrates the educational services [10]. The concept of SOA is to develop an interface among different sources of data. Based on need of service consumers, the services are discovered dynamically.The dynamic services always induce service mechanism, publish, find and bind pattern. It also helps to dynamically, add, remove, replace and relocate the services without doing any modification. Consider instances, load balancing as a services to repair the faulty systems. Reference architecture is mostly used for enabling the rapid deployment of technology.

The presentation of software architecture defines all properties of decision making process. Based on design of architecture, the variant stakeholders present interest in architecture. The documentation of the system might vary according to the communication systems.Some of the documents may define theset of operations using technical compatibility.It affects the attribute quality of the systems.The fig.2.1 presents the service oriented model for Personalized E-learning System 


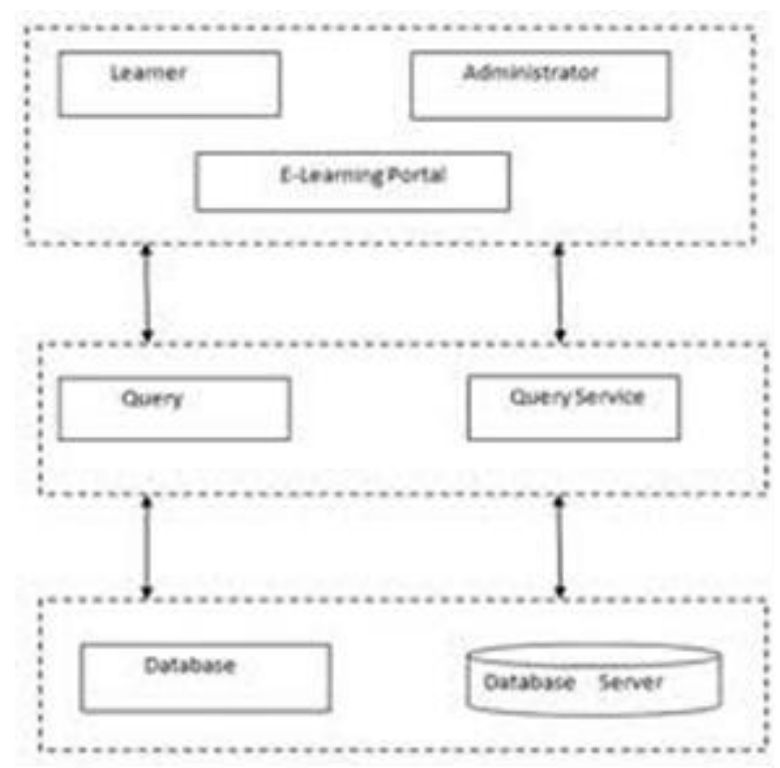

Fig.2.1 Service oriented reference architecture for personalized e-learning systems [10]

A) Presentation Layer: It composes of multiple interfaces of all application data. It manages the tier application of the data. Mostly, it won't entitle the presentation model of the systems. Each application team holds the skins, templates and the style sheets.It includes sample application which leverages the portal navigation in horizontal and vertical tabs. The application is personalized by portlet layout and the background template. It also maintains the centralized model which requires multiple logins [11].

B) Service Layer: It performs the task of services to the software layers. It also invokes, discovers and guide the services required for softwares.It specially binds the provider and consumer with different level of composite services. The services are done for service providers and the consumers are done by service consumers. The opt services are coarse-grained, loosely coupled and the standard based systems. The producer of services publishes by service registry during runtime binding process. It also acts as the predefined business policies during runtime systems. The main task of service manager is to manage, monitor and report the services used in business models. Based on enterprise systems, the services are explained.

C) Application layer:It composes of assessment that performs the surveys, formative and summative assessments. This layer mainly composes of storing, retrieving, deleting and updating all the learning functions.Learning management database is used for maintaining and storing the physical files of the course [12]. In similar way, the task of communication module is to administer the sessions in both asynchronous and synchronous systems. It also merges with other domains like conferences, virtual classrooms and sharing process etc. The Interaction module performs file transfer, messaging and publish-subscribe functions.

Based on receiving components, the publisher or subscriber is adopted for e-learning systems. Each output channel holds copy of the message. It allows the receiver to read the messages. It initiates the identification, authentication, access control and the database access. It should always satisfy the security constraints. The function of identification and authentication is to recognize an end user and validate the end user's identity. Each elementcomposes of aggregate number of end users, information types and controls those elements

D) Database layer: The elements contain database of the systems that compromise the application server models. Each database interface assumes as model for learning environment [13]. The main challenge is of database is the sharing of the systems.Learning components describes the data storing process. With the assistance of database layers, the learning components are stored and retrieved. In some cases, relational databases are widely used for designing (or) meeting the learning technologies.

\section{SECURITY IN WEB- LEARNING SYSTEMS \& RESULTS}

The task of security components are authentication, access control and confidentiality. The requests/ response are handled by the authentication management systems [14]. Based on credentials, the certificates are issued. Authentication process follows the authorization. Based on decision given by authentication system, the authorization follows the users or system.In order to access the resource, identity of the users is monitored. Confidentiality [15] is the main factor of security services in untrusted communication networks.Encryption mechanisms are mainly deployed for securing the data.The fig.2.2 presents the security model of the e-learning portals.

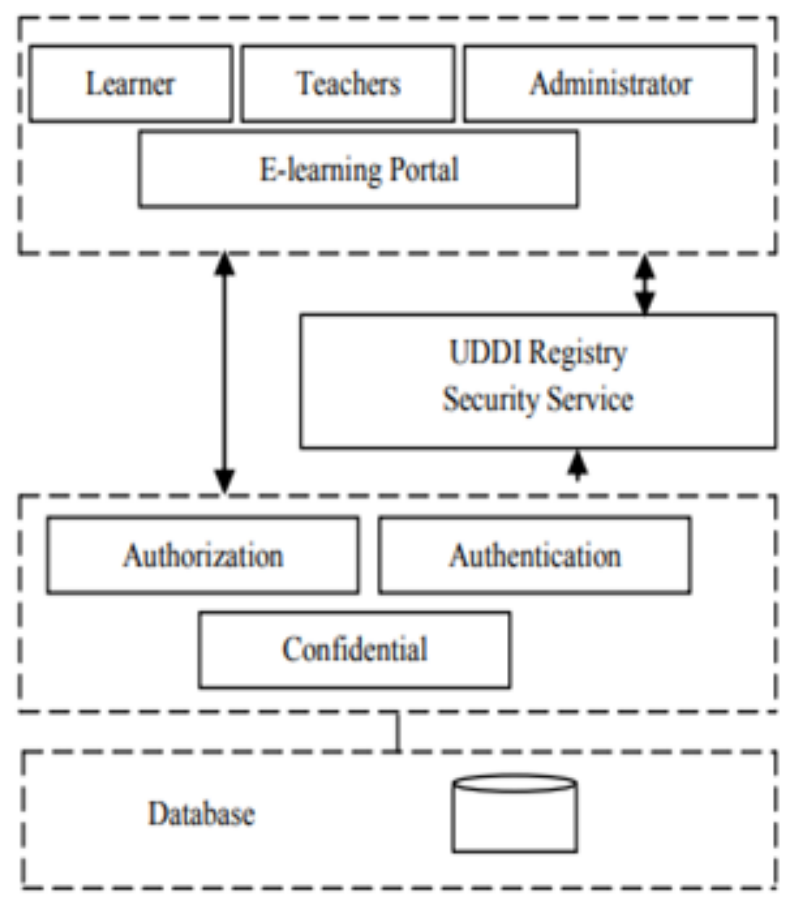

Fig.2.2 Security model of e-learning portal [16]

\subsection{PRIOR TECHNIQUES}

In [17], they presented a case study on e-learning systems.It described the laboratory skills of engineering data. Automatic grading models were been used for

Published By: 
collaborative systems. Though they have predicted the performance systems, yet failed to study on different modalities which lack the independency of the students.In [18], theyexplored the challenges in e-learning systems. The relationship between student and instructors has been to build learning management systems. In addition to, they have also created a better interface for all entities in elearning systems. They explored the demerits faced by the students and instructor for handling the persisted issues.

Ontological basedmemetic agents are studied by [19].Agent system is used to describe the ontological process in structured way. At first, learning path is created for each agent. By doing so, they defined the shortest path for each learning models. They have also elucidated best personalized e-learning experiences. The convergence of local and global search is not aligned for obtained fitness functions. The disengagement of the student in online system is studied by [20]. It is been surveyed that engagement of the students plays a vital part in e-learning systems. Content delivery system is the field of study which imposes several service oriented challenges. HTML tutor system is studied for predicting the e-learning systems.

In addition to, context dimensions is also used for enhancing the TEL process. This systems failed in real time possibilities where reranking of the resources involves high computational complexity. The author in [21] studied about the adaptation strategies. High level adaptation in learner's choice was discovered. The model is designed in two ways, namely, generation adaptation patterns and the strategies followed by the semi-automatic patterns.It generally portrays the strategies employed with existing techniques. Some volunteers have been implemented to discuss about the e-learning domain.

A hypergraph based personalized e-learning process and resource optimization systems model was developed by [22].A personalized learning process was invented to describe the construction process of e-learning systems.It also describes the relationship between process, learners and resources. In [21], the authors presented personalized elearning systems using frog leap algorithms. It introduced personalized learning model using frog-leaping algorithm. It combined with genetic and particle swarm optimization model. It has been experimented on no.of learners which yielded higher convergence rate.

European educational systems were studied by [22] that surveys online and offline courses.Software is designed to analyze and take the decision for each learner.It extended the educational courses for each outcomes of learning. The visualization of learning analytics is done for dashboards at different levels of perspectives.In [23],the author studied dynamic learning style using pattern recognition techniques.Learning styles are predicted by its pattern recognition models. Some of the learning styles determine variant learning topics with appropriate learning prediction outcomes.

Student modelling systems is studied by [24] OSSM interfaces and models are been used for assessing large scale systems. In some cases, the non-learning content makes use of database management systems.A recommendation system was developed by [25] using web 2.0 tools. Similar designed to find the gap. Then, an interface is been designed

learner's algorithms were developed based on learner's requirements.

\begin{tabular}{|l|l|}
\hline \multicolumn{1}{|c|}{ FACTORS of } & WEB BASED LMS \\
\hline $\begin{array}{l}\text { Quality } \\
\text { information }\end{array}$ & Improper updates \\
\hline $\begin{array}{l}\text { Quality of the } \\
\text { system }\end{array}$ & Better in efficiency \\
\hline $\begin{array}{l}\text { Quality of the } \\
\text { service }\end{array}$ & Lack of Scalability \\
\hline Subjective form & $\begin{array}{l}\text { Content alignment needs } \\
\text { approval }\end{array}$ \\
\hline Self- efficacy & Content updation \\
\hline
\end{tabular}

Table 1. Analysis on current factors affecting in web based e-learning systems

\section{CONCLUSION}

In this paper, Data Mining techniques and current state of art for betterment of E-learning system is presented. The survey will be helpful for researchers, business analysts and most importantly for teachers and students. After a detailed study, it is concluded that the most promising approach is Intelligent Adaptive system. It can be helpful in improving the Learning environment. In real environments, machine learning and artificial intelligence techniques with data mining concepts such as classification and clustering algorithms are required. The instructor/course content provider has to make the content of different styles, mentality levels and formats. This method is very expensive to implement and time taking. The main purpose of this term paper is to provide the studies related to the online learning system, by comparative study of previous work in a form of the survey. Data mining provides researcher efficient tools and methods to enhance the decision-making ability and determines the new approaches to implementing personalized learning environment. It is been evaluated that the lack of technical support, improper content updating, insufficient web resources and the security issues degrades the performance of the web based e-learning systems. Our future work will attempts on personalization and security of the web-based e-learning systems using clustering technologies. Initially, the future work is on two phases, namely, personalization of the e-learning contents and the personalized content is further secured by designing efficient algorithms. The designed model will further be evaluated using time and efficiency oriented metrics..

\section{REFERENCES}

1. Konstantinos C. Giotopoulos, et. al., Integrating Agents andComputational Intelligence Techniques in $\mathrm{E}$ LearningEnvironments, Proceedings of World Academy of Science,Engineering and Technology, Volume 7, August 2005.

2. Athanasios D. Styliadis, Ioannis D. Karamitsos, et.al,Personalized e-Learning Implementation - The GIS CaseInternational Journal of Computers, 
Communications \&Control Vol.1, pp. 59-67, 2006.

3. K. Palanivel, S. Kuppuswami Service Versioning Modelfor Personalized E- Learning System, Inter. Journal ofEngineering Sciences \& Technology, Vol.2(10):5583-5593,2010.

4. Job Habraken, Reference Architecture for eLearningSolutions, Master Thesis, Faculty Computer Science, OpenUniversity January, 2008.

5. Job Habraken Reference Architecture for eLearningSolutions, Master Thesis, Open University FacultyComputer Science, 2008

6. AlaeddinKalantari, Designing A Logical Security Framework For Enterprise Service Oriented Architecture (ESOA), Master Thesis, Centre For Advanced Software Engineering (Case), Faculty Of Computer Science And Information System UniversitiTeknologi Malaysia, 2009

7. Athanasios D. Styliadis, Ioannis D. Karamitsos, Personalized e-Learning Implementation - The GIS Case International Journal of Computers, Communications \& Control Vol.1, pp. 59-67, 2006.

8. PanagiotisGermanakos, Nikos Tsianos, et.al., A Semantic Approach of an Adaptive and Personalized Web-based Learning Content - The case of Adaptive Web, Second International Workshop on Semantic Media Adaptation and Personalization,2007.

9. QinghuaZheng, Bo Dong, A Service-oriented Approach to Integration of E-learning Information and Resource Management Systems, IEEE, 2008.

10. Ruben Miguez, Juan M. Santos, and Luis Anido, A Holistic Framework to Support ICT-based Early Childhood Education Processes 39th ASEE/IEEE Frontiers in Education Conference, October 18-21, 2009

11. Mario Munoz organero et al, Personalized ServiceOriented E-Learning Environments, IEEE transactions on Internet Computing, 2010.

12. StylianosSergis et al, Learning Object Recommendations for TeachersBased on Elicited ICT Competence Profiles, IEEE transactions on learning technologies, 9(1), 2016.

13. Peter Brusilovsky et al, Open Social Student Modeling forPersonalized Learning, IEEE transactions on emerging topics in computing, 2015 .

14. AbelardoPardo et al, Combining University Student Selfregulated Learning Indicators and Engagement with Online Learning Events to Predict Academic Performance, , IEEE transactions on learning technologies, 2017.

15. Beatriz Florian-Gaviria et al, A Software Suite for Efficient Useof the European Qualifications Frameworkin Online and Blended Courses, , IEEE transactions on learning technologies, 6(3), 2013.

16. KatrienVerbert et al, Context-Aware Recommender Systems forLearning: A Survey and Future Challenges, IEEE Transactions On Learning Technologies, 5(4), 2012

17. Ben Hanson et al, ReLOAD: Real LaboratoriesOperated at a Distance, IEEE Transactions On Learning Technologies, 2(4), 2009

18. Juan Yang et al, Dynamic Learning Style Prediction MethodBased on a Pattern Recognition Technique, IEEE Transactions On Learning Technologies, 7(2), 2012

19. Giovanni Acampora et al, Exploring e-learning knowledge through ontological agent, IEEE computational magazine, 2010.

20. NatasaHoic-Bozic et al, Recommender System and Web 2.0 Toolsto Enhancea Blended Learning Model, IEEE Transactions on Education, 2015

21. MihaelaCocea and Stephan Weibelzahl, Disengagement Detection in Online Learning:Validation Studies and Perspectives, IEEE Transactions on Learning Technologies, 4(2), 2011.
22. NadjetZemirline et al, Expressing Adaptation StrategiesUsing Adaptation Patterns, IEEE Transactions On Learning Technologies, 5(1),2012

23. Xuedong Sun and Yonghe Lu, Directed-hypergraph based Personalized E-learning Process and Resource Optimization, Fourth International Conference on Digital Home, 2012.

24. M. Gomez-Gonzalez and F.Jurado, Personalized Elearning Using Shuffled Frog-Leaping Algorithm, IEEE transactions on learning systems, 2012.

25. Dan Chiribuca, Daniel Hunyadi, Emil M. Popa, The Educational Semantic Web, International Conference on Applied informatics and Communications, (AIC'08), Greece, 20-22, August 2008. 